\title{
Acción antioxidante conjunta de extractos etanólicos de Mollinedia lanceolata, Croton leptostachyus y Siparuna sessiliflora
}

\author{
Claudia C. Pérez-Jaramillo, Wilmer Fernando Sánchez-Peralta, Walter Murillo-Arango*, John J. Méndez-Arteaga
}

Grupo de Investigación en Productos Naturales-GIPRONUT, Departamento de Química, Facultad de Ciencias, Universidad del Tolima, Barrio Santa Helena Parte Alta, Ibagué, Colombia

\begin{abstract}
Resumen
En los últimos años el daño oxidativo se ha detectado en enfermedades como el cáncer, la diabetes y el Alzheimer, entre otras. Como una alternativa para la prevención y tratamiento de tales enfermedades, se ha propuesto el consumo de antioxidantes, especialmente de fuentes vegetales; sin embargo, se ha estudiado poco el efecto de las mezclas de compuestos de naturaleza antioxidante o de matrices que los contienen. En este trabajo se evaluó el potencial antioxidante de extractos y mezclas de Mollinedia lanceolata (Monimiaceae), Siparuna sessiliflora (Siparunaceae) y Croton leptostachyus (Euphorbiaceae). Se prepararon extractos etanólicos crudos de las tres plantas previamente caracterizados mediante procedimientos de fitoquímmica. Se hicieron pruebas antioxidantes indirectas con el radical 1,1-difenil-2-picrilhidracilo (DPPH) y el catión del ácido 2,2'-azino-bis 3-etilbenzotiazolina-6-sulfónico (ABTS), se determinaron las concentraciones de inhibición media $\left(\mathrm{CI}_{50}\right)$ usadas como base para la preparación de mezclas en una proporción de uno a uno con todos los extractos y se evaluó de nuevo su actividad antioxidante y antihemolítica, así como su índice de combinación con el programa de acceso libre CompuSyn. En los especímenes de estudio se hallaron polifenoles, taninos, flavonoides, terpenos, esteroides y alcaloides. El extracto más activo en la estabilización del radical DPPH fue el de C. leptostachyus $\left(\mathrm{CI}_{50}=53,5 \pm\right.$ $1,1 \mathrm{mg} / \mathrm{l})$ y en la del ABTS, el de M. lanceolata $\left(\mathrm{CI}_{50}=128,8 \pm 0,9 \mathrm{mg} / \mathrm{l}\right)$. Todas las mezclas actuaron de manera sinérgica para estabilizar el DPPH con índices de combinación (IC) mayores de uno, en tanto que en el caso del ABTS las mezclas fueron antagónicas (IC $>1$ ), excepto la de $M$. lanceolata y $S$. sessiliflora $(\mathrm{IC}<1)$. Ninguno de los extractos presentó efecto antihemolítico manifestado en mayores tasas de hemólisis, especialmente en las mezclas. (C) 2017. Acad. Colomb. Cienc. Ex. Fis. Nat.
\end{abstract}

Palabras clave: Actividad antioxidante; Extractos vegetales; Acción conjunta; Sinergismo; Antagonismo; Monimiaceae; Euphorbiaceae; Siparunaceae.

Joint antioxidant action of ethanolic extracts of Mollinedia lanceolata, Croton leptostachyus and Siparuna sessiliflora

\begin{abstract}
In recent years, oxidative damage has been associated with diseases such as cancer, diabetes and Alzheimer, among others. The consumption of antioxidants, especially from vegetable sources, has been proposed as an alternative for prevention and treatment; however, few studies have been conducted on the effect of mixtures of antioxidant compounds or matrices that include them. In this study we used crude extracts and mixtures of extracts from Mollinedia lanceolata, Siparuna sessiliflora and Croton leptostachyus. We prepared crude ethanolic extracts from the three plants previously characterized with phytochemical procedures. We performed indirect antioxidant tests with the DPPH and ABTS radicals, we determine the various $\mathrm{IC}_{50}$ used as the basis for preparing 1:1 mixtures with all the extracts and we reassess their antioxidant and antiemolitic activity, as well as their combination index using the free-access software CompuSyn. We found polyphenols, tannins, flavonoids, terpenes, steroids and alkaloids. The most active extract in the stabilization of the DPPH radical was C. leptostachyus $\left(\mathrm{IC}_{50}=53.5 \pm 1.1 \mathrm{mg} / \mathrm{l}\right)$ and of ABTS, $M$. lanceolata $\left(\mathrm{IC}_{50}=128.8 \pm 0.9 \mathrm{mg} / \mathrm{l}\right)$. All mixtures acted in synergy to stabilize DPPH with combination indices (CI) higher than 1, whereas in ABTS mixtures they were antagonistic (CI $>1)$, except for M. lanceolata + $S$. sessiliflora $(\mathrm{CI}<1)$. None of the extracts presented an antihemolytic effect manifested in higher hemolysis rates, especially in the mixtures. (C) 2017. Acad. Colomb. Cienc. Ex. Fis. Nat.
\end{abstract}

Key words: Antioxidant activity; Plant extracts; Joint action; Synergism; Antagonism; Monimiaceae; Euphorbiaceae; Siparunaceae.

*Correspondencia: Walter Murillo Arango, wmurillo@ut.edu.co; Recibido: 19 de octubre de 2016; Aceptado: 14 de febrero de 2017 


\section{Introducción}

En un intento constante por mejorar la calidad de vida, se han utilizado plantas como fuente de alimento, abrigo, ropa, medicinas y cosméticos; algunas de estas presentan un amplio potencial medicinal, convirtiéndose en el único recurso terapéutico de las comunidades y grupos étnicos especialmente en América del Sur, contribuyendo de manera significativa a la atención primaria en salud (Betoni, et al., 2006), aproximadamente, el $80 \%$ de los habitantes del mundo confían en la medicina tradicional. (Dawoud, et al., 2013). Existen numerosos ejemplos de formulaciones complejas utilizados en la medicina tradicional China y otras medicinas tradicionales en todo el mundo; por esto la idea de combinar dos o más extractos de plantas en una combinación efectiva se remonta muchos siglos. El sinergismo esta basado en la combinacion de metabolitos de dos o mas sustancias que puede resultar en respuestas o interacciones sinérgicas, aditivas o antagonicas entre los componentes combinados en las células u organismos (Efferth, Koch, 2011)

Estos análisis de interacción se han realizado especialmente en fármacos y en menor proporción en plantas o metabolitos secundarios, de esta manera se aprovecha su potencial. por ende la combinación de dos o más sustancias se convierten en un gran acierto, para lograr efectos terapéuticos deseados, potencializando la actividad proporcionada por los agentes individuales, utilizándose entonces en el tratamientos de enfermedades oncológicas e infecciosas (Ren, et al., 2003).

Existen multiples ejemplos actualmente de que la interacción de fármacos aumenta las posibilidades de éxito en el tratamiento y el control de enfermedades como leucemias y linfomas (Glode, Jarkowski, 2009), el cáncer (Chen, et al., 2015) entre otras.

En este trabajo se evaluó el potencial antioxidante de mezclas binarias y terciarias realizadas a partir de extractos vegetales obtenidos de 3 plantas altamente utilizadas por las comunidades indígenas del mundo en la cura de diferentes enfermedades, estas fueron $M$. lanceolata (Solanilla, et al., 2011), S. sessiliflora (Padilla González, Gil Archila, 2012) y C. leptostachyus (Chiappe Pulido, 2013); como estudio preliminar se realizó un tamizaje fitoquímico a los extractos crudos además de evaluar la posible actividad antioxidante de los extractos crudos y sus mezclas (binarias y terciarias) mediante métodos indirectos (DPPH, ABTS), un método directo, evaluando el porcentaje de hemolisis en un modelo de eritrocitos; por último se evaluó el comportamiento presentado por las mezclas de los extractos a través de un modelo de interacción (CompuSyn), el cual permite obtener índices de combinación (IC) y así determinar cuantitativamente las interacciones entre medicamentos o extractos en este caso, donde IC $>1$ indica antagonismo, $\mathrm{IC}=1$ indica aditividad $\mathrm{y}$ IC $<1$ sinergismo.

\section{Metodologia}

Material vegetal. Para este estudio se utilizaron las partes aéreas (hojas) de las 3 plantas objeto de estudio; la recolección de la información etnobotánica y de las muestras vegetales, se hizo en tres municipios; Mollinedia lanceolata (romadizo) se recolecto en el Municipio de Yopal (Casanare), a una altura de $350 \mathrm{msnm}$, entre las coordenadas $05^{\circ} 21^{\prime} 06^{\prime \prime}$ de latitud norte y $72^{\circ} 24^{\prime} 12^{\prime \prime}$ de longitud oeste; Croton leptostachyus (mosquero) fue recolectada en el corregimiento de Payande perteneciente al municipio de San Luis (Tolima), a una altura de $506 \mathrm{msnm}$, entre las coordenadas $4^{\circ} 19^{\prime} 41^{\prime}$ ' Latitud Norte y $75^{\circ} 13^{\prime}$ 22" Longitud Oeste, (Acosta, et al., 2001) Diagnostico para el plan de desarrollo municipal de San Luis 2012- 2015) y Siparuna sessiliflora (limón de monte) se recolecto en la Universidad del Tolima que se encuentra dentro del área urbana del municipio de Ibagué (Tolima), a una altura de $1285 \mathrm{msnm}$, se encuentra en las coordenadas geográficas: $4^{\circ}$ 15 > a N $4^{\circ} 40$ > latitud norte y $75^{\circ} 00$ > a $75^{\circ} 30$ longitud oeste (Vanegas Gálvez, 2002). Para finalizar la identificación de cada una de las tres plantas, se enviaron bouchers de cada una al herbario de la Universidad Nacional, allí fueron identificadas y se les asigno un numero de colección así: M. lanceolata 530879 y C. leptostachyus 572765 y $S$. sessiliflora 575456.

Obtención de los extractos vegetales. El material vegetal se secó en una estufa a una temperatura de $40{ }^{\circ} \mathrm{C}$ durante 48 horas y, posteriormente, se trituró. Las hojas se pusieron separadamente en frascos de vidrio para realizar extracción por maceración, utilizando como solvente etanol (relación 1:10 de material vegetal/solvente) durante 48 horas; los extractos obtenidos se almacenaron a $5{ }^{\circ} \mathrm{C}$ en frascos debidamente rotulados.

Tamizaje fitoquímico de los extractos vegetales. La detección de los principales grupos de metabolitos se realizó mediante ensayos cualitativos individuales para cada grupo químico, según la guía metodológica para la detección rápida de algunos núcleos secundarios y caracterización de una droga cruda, propuesto por Murillo-Perea y MéndezArteaga (2011). Lo anterior mediante reacciones de precipitación y de coloración.

Preparación de mezclas. Para estos ensayos se realizaron mezclas binarias y terciarias de los 3 extractos vegetales. Como parámetro de combinación se usaron las concentraciones medias inhibitorias $\left(\mathrm{CI}_{50}\right)$ obtenidas en la estabilización de los radicales DPPH y ABTS, guardando proporciones 1:1 para cada extracto, tanto en las mezclas binarias como en las ternarias.

\section{Actividad antioxidante invitro}

Actividad inhibitoria del catión radical ABTS. Para evaluar la actividad estabilizante de cada una de las mezclas (binaria o terciaria) de los extractos, se utilizó la metodología descrita por Marquina, et al., (2008); la mezcla se preparó en relación 1:50 (extracto: radical) este procedimiento se realizó por triplicado; posterior se midió el cambio de densidad óptica a $734 \mathrm{~nm}$ después de los primeros 6 minutos de reacción. La actividad estabilizante del ABTS se determinó mediante la ecuación: 


$$
\operatorname{AEABTS}=\left(\frac{\mathrm{A}_{\mathrm{ABTS}}-\mathrm{A}_{6 \mathrm{~min}}}{\mathrm{~A}_{\mathrm{ABTS}}}\right) \times 100
$$

Donde:

AEABTS: Actividad estabilizante del radical ABTS, expresada en porcentaje

$\mathrm{A}_{\mathrm{ABTS}}$ : absorbancia del ABTS antes de agregar la muestra

$\mathrm{A}_{6 \min }$ : Absorbancia de la mezcla reaccionante a los 6 minutos

Como patrón se utilizó Trolox $(0,0312$ a $1 \mu \mathrm{g} / \mathrm{mL})$, el cual fue sometido a las mismas condiciones de ensayo

Actividad inhibitoria sobre el radical DPPH. La capacidad para estabilizar el radical DPPH se realizó siguiendo la metodología propuesta por Braca, et al., (2002), con algunas modificaciones. Una curva patrón se preparó con trolox $(0,0039$ a $0,0625 \mu \mathrm{g} / \mathrm{mL})$, Los valores de actividad antioxidante, a las distintas concentraciones de trabajo, se calcularon mediante la ecuación:

$$
\% \mathrm{CEDPPH}=\left(\frac{\mathrm{A}_{\mathrm{B}}-\mathrm{A}_{\mathrm{M}}}{\mathrm{A}_{\mathrm{B}}}\right) \times 100
$$

Dónde:

CEDPPH: Capacidad estabilizadora del radical DPPH, expresada en porcentaje.

AB: Absorbancia del blanco

AM: Absorbancia de la mezcla reaccionante

Actividad antioxidante en un modelo de células sanguíneas (eritrocitos). Para la obtención de la sangre se utilizó la metodología propuesta por Sánchez en el 2009. El ensayo para estimar el efecto inhibitorio de los extractos sobre la hemólisis se realizó a través del método descrito por Ebrahimzadeh, et al., (2010) con algunas modificaciones, sobre los 3 extractos crudos de las plantas objeto de estudio

El porcentaje de inhibición de hemolisis fue calculado utilizando la siguiente ecuación:

$\%$ de Inhibición de Hemólisis $=\left[\left(\mathrm{A}_{\mathrm{C}}-\mathrm{A}\right) / \mathrm{A}_{\mathrm{C}}\right] \times 100$

Donde Ac es la absorbancia del control negativo y A es la absorbancia de la muestra (con el extracto a cada una de las concentraciones estudiadas). Como control positivo fue utilizado Ácido Ascórbico a la misma concentración de los extractos (en los ensayos donde se utilizó el Ácido Ascórbico como control positivo; este fue preparado al momento del ensayo).

Análisis estadístico. Las diferentes $\mathrm{CI}_{50}$ generadas para todas las pruebas de la actividad antioxidante y hemolítica se obtuvieron mediante análisis de regresión lineal simple, empleando el paquete estadístico STATGRAPHICS Centurion XV (StatPoint, Inc.) a un nivel de significancia de 0,05. Todos los análisis se realizaron por triplicado.

Análisis de interacción mediante isobolograma. Para realizar este análisis se utilizó un software de versión libre CompuSyn, este programa nos permite apreciar de una manera gráfica la interacción de combinaciones de extractos o fármacos; utilizando ecuaciones que proporcionan las bases teóricas para el índice de combinación (IC) y permiten la determinación cuantitativa de las interacciones entre medicamentos $\mathrm{u}$ otras matrices que se mezclen, donde $\mathrm{IC}=1$, indica un efecto aditivo; $\mathrm{IC}<1$, indica sinergia y $\mathrm{CI}>$ 1 indica efecto antagónico. Basado en estos algoritmos, el software ha sido desarrollado para permitir la simulación automatizada de sinergismo y antagonismo en todos los niveles de dosis o efecto.

\section{Resultados y discusión}

Caracterización de los extractos vegetales. En la tabla 1, se encuentran los resultados obtenidos en la caracterización cualitativa de los 3 extractos evaluados (M. lanceolata, $S$. sessiliflora y C.leptostachyus); en las 3 especies se determinó la presencia de metabolitos como polifenoles, taninos, flavonoides y fenilpropanoides, entre otros. La presencia de compuestos de naturaleza polifenólica en las 3 plantas fue abundante, destacando el contenido de estos metabolitos en M. lanceolata, estos compuestos se destacan por generar defensa ante diversos factores como la radicación UV, ataque de animales, de hongos y bacterias (Cooper-Driver, et al., 2007); sin dejar de mencionar que han sido reportados en literatura científica como unos de los principios activos más importante para la estabilización de radicales (Lambert, $\boldsymbol{e t}$ al., 2007; Cadenas, 2000).

Los ensayos realizados para reconocer núcleos de naturaleza alcaloidal fueron positivos para $M$. lanceolata y $S$. sessiliflora, destacando los de tipo morfina, codeína, papaverina, noscapina, entre otros, y del grupo de las anfetaminas y derivados, como reportó Murillo, et al., (2011). Las saponinas encontradas en C.leptostachyus y $S$. sessiliflora, en esta caracterización podrían ser predictivos de la toxicidad que pueden presentar los extractos de estas plantas, lo cual podría limitar su uso en aplicaciones fitoterapéuticas tales como las que se presentan en este estudio.

\section{Actividad antioxidante in vitro}

Actividad estabilizante de los radicales ABTS y DPPH. Los resultados de la actividad estabilizante del radical DPPH y ABTS en extractos crudos, y las diferentes mezclas expresado como $\mathrm{CI}_{50}$ se representan en la tabla 2 .

El tamizaje cualitativo preliminar realizado a los extractos crudos mostró la presencia de metabolitos referenciados en la literatura científica por su capacidad antioxidante. Las $\mathrm{CI}_{50}$ calculadas para las mezclas de los extractos frente a la estabilización del radical DPPH mostraron mayor efectividad, mientras que para el caso del ABTS ocurre lo contrario, generándose efectos antagónicos, excepto para la mezcla binaria $M$. lanceolata + S. sessiliflora. Hay que tener en cuenta que la capacidad antioxidante de una mezcla no viene dada solo por la suma de las capacidades antioxidantes de cada uno de sus componentes; estos compuestos pueden interactuar entre si y producir efectos sinérgicos o inhibitorios. (Echavarria, et al., 2009)

Trabajos como el de Güder, et al., (2012), donde se comparó la actividad antioxidante de extractos y mezclas de Urtica dioica L.y Malva neglecta Wallr con antioxidantes como el BHA, BHT y $\alpha$-tocoferol; comprobaron que en las 
Tabla 1. Tamizaje fitoquímico realizado a los extractos crudos de M. lanceolata, S. sessiliflora y C. leptostachyus

\begin{tabular}{|c|c|c|c|c|}
\hline \multirow[t]{2}{*}{ Núcleo secundario } & \multirow[t]{2}{*}{ Prueba } & \multicolumn{3}{|c|}{ Extracto } \\
\hline & & M. lanceolata & S. sessiliflora & C. leptostachyus \\
\hline \multirow[t]{2}{*}{ Saponinas } & Espuma & - & + & + \\
\hline & Rosenthaler & - & + & + \\
\hline \multirow{2}{*}{ Polifenoles } & Folin-Ciocalteau & + & + & + \\
\hline & $\mathrm{CCD}$ & + & + & + \\
\hline \multirow[t]{3}{*}{ Taninos } & $\mathrm{FeCl}_{3}$ & + & + & + \\
\hline & Gelatina-sal & + & + & + \\
\hline & Taninos condensados & + & + & - \\
\hline \multirow{2}{*}{ Flavonoide } & Shinoda & + & + & + \\
\hline & PEW'S & + & + & + \\
\hline Fenilpropanoides & Arnow & + & - & + \\
\hline \multirow[t]{2}{*}{ Terpenos y esteroides } & Lieberman-Burchard & + & + & + \\
\hline & Salkowski & + & + & + \\
\hline Iridoides & Vainillina etanólica & - & - & + \\
\hline \multirow[t]{5}{*}{ Alcaloides } & Dragendorff & + & + & - \\
\hline & Mayer & - & + & - \\
\hline & Erlich & - & - & - \\
\hline & Tanred & + & + & - \\
\hline & Valser & + & - & - \\
\hline Quinonas & Bornträger & - & - & - \\
\hline Cumarinas & $\mathrm{CCD}$ & - & + & + \\
\hline Lactonas & $\mathrm{CCD}$ & - & + & + \\
\hline \multirow[t]{2}{*}{ Cardiotónicos } & $\mathrm{CCD}$ & - & + & + \\
\hline & Barfoed & - & + & + \\
\hline
\end{tabular}

+ Presente

- Ausencia bajo las condiciones de la prueba

Tabla 2. Concentraciones inhibitorias medias para la estabilización del radical DPPH y ABTS de los 3 extractos las plantas evaluadas y sus mezclas

\begin{tabular}{lcc}
\hline Plantas y mezclas de las plantas & DPPH & ABTS \\
\hline M. lanceolata & $128,8 \pm 0,9$ & $11,2 \pm 0,3$ \\
C. leptostachyus & $53,5 \pm 1,1$ & $11,6 \pm 0,3$ \\
S. sessiliflora & $304,1 \pm 3,7$ & $47,2 \pm 2,5$ \\
M. laceolata + C. leptostachyus & $13,1 \pm 0,3(\mathrm{IC}<1)$ & $12,0 \pm 0,2(\mathrm{IC}>1)$ \\
M. lanceolata + S. sessiliflora & $99,2 \pm 0,9(\mathrm{IC}<1)$ & $26,8 \pm 0,6(\mathrm{IC}<1)$ \\
C. leptostachyus + S. sessiliflora & $178,1 \pm 2,7$ (IC $<1)$ & $36,7 \pm 1,1(\mathrm{IC}>1)$ \\
M. lanceolata + C. leptostachyus + S. sessiliflora & $80,4 \pm 1,9$ (IC $<1)$ & $27,9 \pm 0,1(\mathrm{IC}>1)$ \\
Trolox (patron) & $3,15 \pm 10^{-2}$ & $5,82 \pm 10^{-1}$ \\
\hline
\end{tabular}

$\pm=$ desviación estándar, IC = Índice de combinación IC $>1=$ antagonismo, $\mathrm{IC}=1$ aditividad, $\mathrm{IC}<1=$ sinergismo

mezclas de estas plantas se encuentran comúnmente acción sinérgica mejorando así su actividad antioxidante. Mïsan, et al., (2011) encontraron efectos sinérgicos en la mezcla obtenida de perejil, espino amarillo, menta y alcaravea; exhibiendo una alta actividad antioxidante en la mayoría de las pruebas (directas o indirectas), realizadas en el estudio.

Actividad antioxidante en un modelo de celulas sanguíneas (eritrocitos). Los resultados observados en la evaluación de la actividad antioxidante demuestran que los extractos crudos y las mezclas de los extractos inhiben especies radicalarias sintéticas como DPPH y ABTS, pero al determinar la actividad hemolítica se observaron efectos diferentes. Los 3 extractos etanólicos crudos de las tres plantas mostraron alta toxicidad frente a las células sanguíneas evaluadas, lo cual se demostró a través de la hemolisis. En los extractos de $C$. leptostachyus y $S$. sessiliflora se explica por la presencia de saponinas, detectadas en el tamizaje preliminar realizado a los extractos (Tabla 1), las cuales podrían enmascarar el potencial protector del resto de metabolitos presentes en los extractos. 
En orden decreciente de toxicidad encontrada fue, $S$. sessiliflora, C. leptostachyus y M. lanceolata. En el caso de $M$. lanceolata, llama la atención debido a que a pesar de no detectarse saponinas en el extracto se observó efecto hemolítico sobre los eritrocitos humanos; por lo tanto la ruptura de estas células puede generarse por posible competencia entre los mecanismos de estabilización y la generación de especies prooxidantes (Pérez, 2003), factor determinante al momento de establecer la estabilidad de las especies generadas y su contribución con respecto a la actividad antioxidante total en un sistema como el que se evaluó en este trabajo.

Los resultados de los análisis de varianza realizados con los extractos de las plantas se pueden apreciar en la tabla 3 .

Cabe resaltar que con este ensayo se cualifico de manera indirecta su toxicidad demostrando que estos extractos a pesar de tener una capacidad para estabilizar los radicales en ensayos indirectos, no serían de utilidad en un sistema directo generando una lisis de la membrana de los eritrocitos, no obstante es necesario realizar estudios más rigurosos para establecer la toxicidad en contraste con el potencial terapéutico, por ejemplo citotóxico de las especies estudiadas.

Hiruma, et al., (2013) investigaron los efectos antitumorales in vitro, para esto utilizaron una mezcla de seis hierbas medicinales que contenían laca. La mezcla de estos extractos inhibió la proliferación de varias líneas tumorales humanas en ratón; demostrándose un potencial sinérgico entre las plantas que se mezclaron. Este tipo de mezclas también se han utilizado para el control de algunas bacterias como Enterobacter aerogenes, Escherichia coli, Salmonella typhi y Staphylococcus aureus, este es el caso del estudio realizado por Vásquez, et al., (2014), quienes mezclaron extractos etanólicos de Larrea tridentata, Flourensia cernua y Opuntia ficus-indica, la mezcla realizada por los extractos inhibió el crecimiento de las bacterias indicando así el efecto sinérgico de sus componentes.

Análisis de interacción mediante isobologramas. Mediante los análisis de interacción realizados con el software CompuSyn se obtuvieron isobologramas que permitieron observar los índices de interacción para cada una de las mezclas, en cuanto a los índices de combinación encontrados para la evaluación del DPPH se hallaron índices inferiores a 1 en las 3 mezclas binarias y la terciaria, destacando la mezcla obtenida a partir de los extractos de $C$. leptostachyus + M. lanceolata, la cual mostró los índices de interacción más efectivos con valores que oscilaban entre 0.02883 y 0.04715 , generándose entonces una interacción sinérgica para la estabilización de este radical; en cuanto a los índices de interacción obtenidos en la mezcla ternaria estos oscilan entre 0.11801 y 0.07813 , índices inferiores a 1 demostrando así una acción sinérgica disminuida con tendencia a la aditividad.

En caso del radical sintético ABTS, los IC hallados en las mezclas binarias y terciaria fueron superiores a 1, lo que indica una interacción de tipo antagónico en todas
Tabla 3. Concentraciones hemolíticas medias $\left(\mathrm{Ch}_{50}\right)$ para la hemolisis de células eritrocitos humanos

\begin{tabular}{lccc}
\hline \multirow{2}{*}{ Especies } & $\mathbf{C h}_{\mathbf{5 0}}(\mathbf{m g} / \mathbf{L})$ & \multicolumn{2}{c}{ Intervalos de Confianza } \\
\cline { 2 - 4 } & Media & Inferior & Superior \\
\hline M. lanceolata & $65,3 \pm 0,2$ & 62,9 & 67,7 \\
C. leptostachyus & $85,9 \pm 0,1$ & 84,4 & 87,4 \\
S. sessiliflora & $92,8 \pm 0,1$ & 91,5 & 94,1 \\
\hline
\end{tabular}

las mezclas evaluadas. La mezcla binaria $M$. lanceolata + S. sessiliflora, muestra los IC más altos, estos oscilaron entre 0.74468 y 211.001 . En cuanto a la mezcla ternaria se evidenciaron valores entre 18.4807 y 2.23341 , evidenciando una fuerte acción antagónica.

Los resultados de interacción obtenidos para la estabilización de los dos radicales fueron diferentes por lo que es importante considerar la variabilidad en los mecanismos de estabilización radicalaria y la interacción que puede existir entre los distintos metabolitos que influencian la actividad antioxidante, ya que la mezcla de extractos o componentes bioactivos no necesariamente potencian una determinada actividad biológica. En este caso las mezclas generaron interacciones que no están relacionadas exactamente con la actividad individual de sus componentes, por esto se hace necesario realizar este tipo de evaluaciones. Los resultados indican que las mezclas favorecen la estabilización de radicales libres vía transferencia de átomos de hidrogeno, no obstante si se considerada la estabilización por trasferencia de electrones el efecto es contrario.

Hay que resaltar que este tipo de estudios son poco comunes en matrices vegetales, más cuando se pueden contrastar con un modelo de evaluación directa como el que representa la membrana eritrocitaria. Actualmente la combinación de fármacos es la más ampliamente utilizada en el tratamiento de las enfermedades, como el cáncer y el SIDA. Los principales objetivos son lograr un efecto terapéutico sinérgico, reducir la toxicidad, y minimizar o retrasar la inducción de resistencia a los medicamentos (Chou, 2006); otra de las combinaciones usadas son las que se generan entre alimentos que contienen nutrimentos y otras sustancias que pueden afectar en diferente grado la farmacocinética y la farmacodinamia de los medicamentos $\mathrm{y}$, de igual manera, los medicamentos que causan impacto sobre el estado nutricional, ya sea produciendo una deficiencia nutricional o interfiriendo en el proceso natural de la alimentación (Quir, 2014).

En este sentido se han combinado diferentes tipos de vegetales como por ejemplo el tomate, la zanahoria, la berenjena y la patata violeta; para evaluar la interacción antioxidante frente a radicales sintéticos, dichos ensayos mostraron efectos antioxidantes sinérgicos en combinaciones binarias, muy similar a lo encontrado en el estudio actual (Jiang, et al., 2015). Otra manera de aumentar el potencial se los antioxidantes naturales como él te verde 
es generar una interacción con antioxidantes sintéticos como el ácido ascórbico, como lo hicieron Enko, et al., (2015); al realizar una serie de combinaciones entre estos dos antioxidantes comprobando su capacidad a través de diferentes ensayos indirectos y donde se determinó un tipo de interacción potencializada.

\section{Conclusiones}

Los extractos etanólicos de 3 plantas estudiadas demostraron ser fuentes ricas en compuestos fenólicos y al mismo tiempo expusieron capacidad para la estabilización de los radicales sintéticos DPPH y ABTS, no obstante las mezclas presentaron comportamientos que dependieron del tipo de combinación realizada, pero primando la acción estabilizadora del radical DPPH en la mayoría de los casos, posiblemente porque los metabolitos presentes favorecen estabilización radicalária vía transferencia de átomos de hidrogeno. La evaluación de la actividad antioxidante en eritrocitos demostró la competencia que ejercen los metabolitos al estar en una mezcla compleja, que implica el uso de un extracto o preparación de diferentes plantas con fines terapéuticos, por lo que deben realizarse estudios de esta naturaleza que puedan validar el uso seguro de formulaciones que impliquen el uso de extractos o sus mezclas no solo fines antioxidantes sino para cualquier tipo de actividad biológica que se persiga.

\section{Conflicto de intereses}

Los autores declaran no tener conflicto de intereses.

\section{Referencias}

Acosta, R., Gómez, T. J., Morales, G. J., Gómez, N. (2001). Esquema de Ordenamiento Territorial. Alcaldía Municipal de San Luis. Ibagué, Tolima

Akladios, F. N., Andrew, S. D., \& Parkinson, C. J. (2015). Selective induction of oxidative stress in cancer cells via synergistic combinations of agents targeting redox homeostasis. Bioorga. Med. Chem. 23 (13): 3097-3104.

Betoni, J. E. C., Mantovani, R. P., Barbosa, L. N., Di Stasi, L. C., \& Fernandes Junior, A. (2006). Synergism between plant extract and antimicrobial drugs used on Staphylococcus aureus diseases. Memorias do Instituto Oswaldo Cruz. 101 (4): 387-390.

Braca, A., Sortino, C., Politi, M. (2002). Anti-oxidant activity of flavonoids from Licania licaniaeflora. J. Ethnopharmacol., Vol. 792, pp. 379-381.

Calderón, J. C., Fernández, A. Z., de Jesús, A. I. M. (2008). Ateroesclerosis, estrés oxidativo y actividad física. Revisión. Investigación Clínica. 49 (3).

Camacho, K. M., Kumar, S., Menegatti, S., Vogus, D. R., Anselmo, A. C., \& Mitragotri, S. (2015). Synergistic Antitumor Activity of Camptothecin-Doxorubicin Combinations and their Conjugates with Hyaluronic Acid. J. Controlled. Release. 210: 198-207

Caracterización del sector educativo municipio de Yopal. (2012). Secretaria de educación y cultura. Yopal - Casanare

Chen, J., Lin, L., Guo, Z., Xu, C., Tian, H., Park, K., \& Chen, X. (2015). Synergistic treatment of cancer stem cells by combinations of antioncogenes and doxorubicin. J. Drug. Deliver. Sci. Tech. 26: 407-418
Chen, Q. H., Yu, K., Zhang, X., Chen, G., Hoover, A., Leon, F \& Rakotondraibe, L. H. (2015). A new class of hybrid anticancer agents inspired by the synergistic effects of curcumin and genistein: Design, synthesis, and antiproliferative evaluation. Bioorga. Med. Chem. Letters. 25 (20): 4553-455;

Chiappe, P.A. C. (2013). Estudio fitoquímico de las hojas de la especie vegetal Croton schiedeanus (Euphorbiaceae) (Doctoral dissertation, Universidad Nacional de Colombia).

Chou, T. C. (2006). Theoretical basis, experimental design, and computerized simulation of synergism and antagonism in drug combination studies. Pharmacol. Rev. 58 (3): 621-681

Cooper-Driver, G. A., Bhattacharya, M. (1998). Role of polyphenolics in plant evolution. Phytochem. 49: 1165-1174.

Dawoud, M. E. A., Mawgoud, Y. A., Dawoud, T. G. (2013). Synergistic interactions between plant extracts, some antibiotics and/or their impact upon antibiotic-resistant bacterial isolates. Afric. J. Biotechnol. 12 (24): 3835

De Castro, J., Rodríguez, M.C., Martínez-Zorzano, V.S., Hernández-Hernández, A., Llanillo, M. y SánchezYagüe, J. (2008). Erythrocyte and platelet phospholipid fatty acids as markers of advanced non-small cell lung cancer: comparison with serum levels of sialic acid, TPS and Cyfra. 21-1.

Diagnóstico para el plan de desarrollo municipal de San Luis 2012- 2015.

Echavarria Z. B., Franco S. A., Martínez M. A. (2009). Evaluación de la actividad antioxidante y determinación del contenido de compuestos fenólicos en extractos de macroalgas del Caribe Colombiano. Vitae [online]. 16 (1): 126-131.

Efferth, T., Koch, E. (2011). Complex interactions between phytochemicals. The multitarget therapeutic concept of phytotherapy. Curr. Drug Targ. 12: 122-132

Enko, J., Gliszczyńska-Świgło, A. (2015). Influence of the interactions between tea (Camellia sinensis) extracts and ascorbic acid on their antioxidant activity: analysis with interaction indexes and isobolograms. Food. Addit. Contam. Part A. 32 (8): 1234-1242.

Glode, A. E., Jarkowski, A. (2009). Bendamustine: a new treatment option for chronic lymphocytic leukemia. Pharmacotherapy. J. Hum. Pharmacol. Drug Therapy. 29 (11): 1375-1384.

Güder, A., Korkmaz, H. (2012). Evaluation of in-vitro antioxidant properties of hydroalcoholic solution extracts Urtica dioica L., Malva neglecta Wallr. and their mixture. Iran. J. Pharm. Res. 11 (3): 913-923.

Hiruma, W., Suruga, K., Kadokura, K., Tomita, T., Sekino, Y., Komatsu, Y., Kimura, M., Ono, N. (2013). The Antitumor Effects of a Plant Extract Mixture. Pharm. Soc. Jap. 133 (5): 487-491.

Holdridge, L. R. (1987). Ecología basada en zonas de vida (No. 83). Agroamérica.

Jiang, H. W., Yu, C. W., Yang, T. T., Hu, J. N., Liu, R. (2015). The evaluation of antioxidant interactions among 4 common vegetables using isobolographic analysis. J. Food. Sci. 80 (6): 1162-1169

Lambert, J. D., Sang, S., Yang, C. S. (2007). Possible Controversy over Dietary Polyphenols: Benefits vs Risks. Chem. Res. Toxicol. 20: 583-585.

Marquina, V., Araujo, L., Ruíz, J., Rodríguez-Malaver, A., Vit, P. (2008). Composición química y capacidad antioxidante en fruta, pulpa y mermelada de guayaba (Psidium guajava L.). Archivos Latinoamericanos de Nutrición. 58 (1): 98-102. 
Murillo, E., Lombo, O., Méndez, J. (2011). Química y Funcionalidad Biológica de Mollinedia lanceolata (Monimiaceae). Información tecnológica. 22 (2): 3-14

Murillo-Perea, E., Mendez-Arteaga, J. (2011). Guia metodológica para la detección rápida de algunos núcleos secundarios y caracterización de una droga cruda. Sexta revisión. Universidad del Tolima. Facultad de Ciencias. Departamento de Química. GIPRONUT.

Osorio, L. C., Idea, S. (2002). Agenda Ambiental Municipio de Ibagué

Quir, R. E. M. (2014). Fármaco-nutrimento: interacción insuficientemente considerada. Rev Esp Méd Quir. 19: 244-250.

Ren, W., Qiao, Z., Wang, H., Zhu, L., Zhang, L. (2003). Flavonoids: promising anticancer agents. Med. Res. Rev. 23 (4): 519-534.

Stevenson, D. E., Hurst, R. D. (2007). Polyphenolic phytochemicals- just antioxidant or much more. Cell. Mol. Life. Sci. 64 (22): 2900-2916.
Solanilla Duque, J. F., Lombo, O., Murillo Perea, E., Méndez Arteaga, J. J. (2011). Valoración del potencial antioxidante de Mollinedia lanceolata (romadizo). Revista Cubana de Plantas Medicinales. 16 (2): 151-163.

Teuschler, L., Klaunig, J., Carney, E., Chambers, J., Connolly, R., Gennings, C., Giesy, J., Hertzberg, R., Klaassen, C., Kodell, R., Paustenbach, D., Yang, R. (2002). Support of science-based decisions concerning the evaluation of the toxicology of mixtures: a new beginning. Regul. Toxicol. 36: 34-39.

Vanegas Gálvez, M. (2002). Estudio complementario del caso Ibagué, Colombia. In Estudio complementario del caso Ibagué, Colombia. CEPIS

Venereo Gutiérrez, J. R. (2002). Daño oxidativo, radicales libres y antioxidantes. Revista Cubana de medicina militar. 31 (2): 126-133. 